\title{
Thyroid cancer-related bone metastases: increasingly good prospects for treatment
}

\author{
Leonidas H. Duntas ${ }^{1}$
}

Received: 2 March 2018 / Accepted: 21 April 2018 / Published online: 19 May 2018

(c) Springer Science+Business Media, LLC, part of Springer Nature 2018

\begin{abstract}
Medicine starts with the patient, continues with the patient and ends with the patient.
\end{abstract}

Sir William Osler, 1849-1919

The incidence of bone metastases (BM) from differentiated thyroid cancer (DTC) is estimated to be as much as $13 \%$, depending on the type of cancer, being much less common in papillary thyroid cancer than in follicular cancer, of which they are a frequent complication. The latter outcome is due both to the tumor's tendency to spread hematogenously, this being sustained by the synthesis of adhesion molecules from follicular tumor cells that enable them to anchor to the bone matrix, and to the resulting increased blood flow to the bone marrow [1]. Moreover, patient age is also a major factor for BM as they occur mostly in patients over 45 years old [2]. BM have a predilection for the spine, the pelvis, the long bones, the ribs and the base of the skull [3].

Radioiodine (RAI) treatment in DTC patients whose lesions concentrate 131I has long been known to considerably enhance survival rates. Adverse outcome is principally related to the occurrence of skeletal-related events (SREs), defined as spinal cord compression and/or pathological fractures. On the other hand, SREs occurring in patients with thyroid cancer complicated by bone metastases still constitute a poor prognostic indicator, also by comparison with patients suffering from other distant metastases [4]. Currently, a number of modern therapeutic modalities, such as embolization, radiofrequency ablation,

Leonidas H. Duntas

ledunt@otenet.gr

1 Evgenideion Hospital, Unit of Endocrinology, Diabetes and Metabolism, Thyroid Section, University of Athens, Athens, Greece or high-intensity focused ultrasound, can be applied as palliative treatment of BM, though the efficacy and safety of the procedure should be carefully weighed up in the interest of providing optimal treatment [5]. Selective embolization with N-butyl cyanoacrylate for BM from different primary tumors was performed in 243 patients with 309 embolizations [5]. A complete occlusion of metastatic blood supply and more than $80 \%$ devascularization of the lesions were observed in all the lesions, accompanied, however, by considerable side effects in $37 \%$ of the patients [6]. Thus, given that patients with BM from DTC are at especially high risk, the extent of initial treatment and the scheduling of subsequent monitoring need to be meticulously tailored [7]. The recent publication in this Journal of the 2017 retrospective, multicenter M.O.S.CA.TI (Metastases Of the Skeleton from CArcinoma of the ThyroId) study highlights the significance of RAI use in real-life outcome and management of patients with BM while offering hope that this kind of treatment may decrease mortality in patients with SREs and radioiodine-avid BM [8]. Specifically, in 143 patients (63 M, $80 \mathrm{~F}$; median age 64 years) with DTC, 32 with BM, a total of $131 / 143$ patients received RAI treatment. Surgical and/or external beam radiation treatment was undertaken in $47.6 \%$ (68 pat.), while $22.4 \%$ (32 pat.), mostly patients with RAI refractory BM, received antiresorptive bone-acting drugs (zolendronate $=31$, denosumab =1). The duration of follow-up was 48 months and SREs were diagnosed in 53 patients (37.1\%).

The primary end-point of the above study was to appraise current therapeutic approaches to BM in real-life clinical practice as well as to evaluate a number of therapies applied for the prevention of SREs. It was observed that in 24/53 patients, the SREs developed approximately 6 months after BM diagnosis. Meanwhile, in the majority (29/53), the SREs arose during the 48-month observation period, this phenomenon being linked to metachronous BM, localization to the cervical spine and absence of avid RAIuptake. It is noteworthy that those patients with 
metachronous metastases, as compared to synchronous metastases, more frequently had Huerthle cell carcinoma than follicular thyroid carcinoma. Finally, 39 patients died as a result of the development of SREs and localization of BM to the hip.

A major finding of this study is that a statistically significant decrease of overall mortality was achieved only by RAI treatment, by contrast to bone-active drugs and external radiotherapy and even surgery, none of which exerted any significant effects. RAI treatment was administered to 114 patients with metastatic lesions that were radioiodineavid and to 17 with lesions refractory to RAI uptake. Despite the limitations due to the character of the study design, the results are of importance; moreover, they also partially compensate for the lack of any prospective, randomized, controlled studies for the efficacy of 131I in the treatment of BM from DTC. What data exist are retrospective. For instance, in a study investigating survival among 444 patients with distant metastases, those with BM had a 20 -year survival rate of $8 \%$, without any impact on the individual course of disease by bone metastasis, which was later detected [9]. In patients younger than 45 years old, compared to those older than 45 , higher rates of total or partial remission were demonstrated, suggesting a curative effect of 131I in younger patients who have solitary lesions when this is accompanied by surgical extirpation of bone metastases [10].

This study by Mazziotti et al. documented delayed treatment initiation with antiresorptive drugs in real life, this approach usually being considered only when development of SREs has occurred or in patients with BM recalcitrant to RAI. Thus, the non-timely administration of such drugs may account for their reported failure to control the progression of disease. Denosumab, the antibody-mediated antiresorptive agent used to block bone resorption pathways, was applied in only one case and has therefore not been evaluated. However, denosumab, targeting receptor activator for nuclear factor kappa-B ligand of which the baseline levels are potential predictors of bone response, should be considered as an early treatment option in these subjects at high risk for bone events, particularly given that the latter patients are usually under TSH suppressive treatment with 1-thyroxine, which is known to trigger bone loss. Denosumab can also be combined with thyrosine kinase inhibitors in an attempt to block progression of bone metastases, that are often refractory, possibly avoiding SREs in RAI recalcitrant BM. Fortunately, other emerging drugs such as inhibitors of sclerostin, an osteocyte-produced Wnt signaling pathway antagonist, dual-action agents such as proteasome inhibitors that activate osteoblasts and inhibit osteoclasts, or compounds including romosozumab which target osteoblasts thereby activating bone formation, could soon be efficaciously combined with RAI in BM and SREs $[11,12]$.

The survey by Mazziotti et al. of the M.O.S.CA.TI study is a truly important one in the medical literature, clearly illustrating as it does the successful use of RAI treatment in real-life management of DTC-related BM as well as SREs, a method which can substantially decrease the cancer mortality rate as well as considerably lighten the healthcare burden. This highly informative study should therefore encourage the design of targeted RAI therapy that maximizes treatment efficacy by utilizing multimodal imaging techniques within the concept of theranostics, thus promoting precision-guided cancer treatment [13].

Finally, the study results, while variable, are expected, through further investigation and analysis, to shed light on the way forward to identifying new pathways in the treatment of these life-threatening entities.

\section{References}

1. J. Wexler, Bone metastases from differentiated thyroid carcinoma. in Thyroid Cancer, ed. by L. Wartofsky, D. Van Nostrand (New York: Springer, 2016), pp. 723-733)..

2. M. Schlumberger, C. Challeton, F. De Vathaire, J.P. Travagli, P. Gardet, J.D. Lumbroso, C. Francese, F. Fontaine, M. Ricard, C. Parmentier, Radioactive iodine treatment and external radiotherapy for lung and bone metastases from thyroid carcinoma. J. Nucl. Med. 37, 598-605 (1996)

3. A.C. Chiu, E.S. Delpassand, S.I. Sherman, Prognosis and treatment of brain metastases in thyroid carcinoma. J. Clin. Endocrinol. Metab. 82, 3637-3642 (1997)

4. P. Choksi, M. Papaleontiou, C. Guo, F. Worden, M. Banerjee, M. Haymart, Skeletal complications and mortality in thyroid cancer: a population-based study. J. Clin. Endocrinol. Metab. 102, 1254-1260 (2017). https://doi.org/10.1210/jc.2016-3906

5. A.F. Mavrogenis, A. Angelini, C. Vottis, E. Pala, T. Calabrò, P.J. Papagelopoulos, P. Ruggieri, Modern palliative treatments for metastatic bone disease: awareness of advantages, disadvantages, and guidance. Clin. J. Pain. 32, 337-350 (2016). https://doi.org/ 10.1097/AJP.0000000000000255

6. G. Rossi, A.F. Mavrogenis, E. Rimondi, L. Braccaioli, T. Calabrò, P. Ruggieri, Selective embolization with N-butyl cyanoacrylate for metastatic bone disease. J. Vasc. Interv. Radiol. 22, 462-470 (2011). https://doi.org/10.1016/j.jvir.2010.12.023

7. B.H. Lang, K.P. Wong, C.Y. Cheung, K.Y. Wan, C.Y. Lo, Evaluating the prognostic factors associated with cancer-specific survival of differentiated thyroid carcinoma presenting with distant metastasis. Ann. Surg. Oncol. 4, 1329-1335 (2013). https:// doi.org/10.1245/s10434-012-2711-x

8. G. Mazziotti, A.M. Formenti, M.B. Panarotto, E. Arvat, A. Chiti, A. Cuocolo, M.E. Dottorini, C. Durante, L. Agate, S. Filetti, F. Felicetti, A. Filice, L. Pace, T. Pellegrino, M. Rodari, M. Salvatori, C. Tranfaglia, A. Versari, D. Viola, S. Frara, A. Berruti, A. Giustina, R. Giubbini, Real-life management and outcome of thyroid carcinoma-related bone metastases: results from a nationwide multicenter experience. Endocrine 59, 90-101 (2018). https://doi.org/10.1007/s12020-017-1455-6

9. C. Durante, N. Haddy, E. Baudin, S. Leboulleux, D. Hartl, J.P. Travagli, B. Caillou, M. Ricard, J.D. Lumbroso, F. De Vathaire, M. Schlumberger, Long-term outcome of 444 patients with distant 
metastases from papillary and follicular thyroid carcinoma: benefits and limits of radioiodine therapy. J. Clin. Endocrinol. Metab. 8, 2892-2899 (2006)

10. Z.L. Qiu, H.J. Song, Y.H. Xu, Q.Y. Luo, Efficacy and survival analysis of 131I therapy for bone metastases from differentiated thyroid cancer. J. Clin. Endocrinol. Metab. 96, 3078-3086 (2011). https://doi.org/10.1210/jc.2011-0093

11. M.T. Drake, J.N. Farr, Inhibitors of sclerostin: emerging concepts. Curr. Opin. Rheumatol. 26, 447-452 (2014). https://doi.org/10. 1097/BOR.0000000000000073
12. S. Sousa, P. Clézardin, Bone-targeted therapies in cancer-induced bone disease. Calcif. Tissue Int. 102, 227-250 (2018). https://doi. org/10.1007/s00223-017-0353-5

13. H. Jadvar, Targeted radionuclide therapy: An evolution toward precision cancer treatment. Ajr. Am. J. Roentgenol. 209, 277-288 (2017). https://doi.org/10.2214/AJR.17.18264 